\title{
Existence, Uniqueness, and Input-to-State Stability of Ground State Stationary Strong Solution of a Single-Species Model via Mountain Pass Lemma
}

\author{
Ruofeng Rao $\mathbb{D}^{1,2}$ Quanxin Zhu $\mathbb{D}^{3},{ }^{3}$ and Jialin Huang $\mathbb{C}^{4,5}$ \\ ${ }^{1}$ School of Mathematical Sciences, Sichuan Normal University, Chengdu 610066, China \\ ${ }^{2}$ Department of Mathematics, Chengdu Normal University, Chengdu 611130, China \\ ${ }^{3}$ School of Mathematics and Statistics, Hunan Normal University, Changsha 410081, China \\ ${ }^{4}$ Department of Mathematics, Sichuan Sanhe College of Professional, Luzhou 646200, China \\ ${ }^{5}$ School of Information Science and Engineering, Chengdu University, Chengdu 610106, China \\ Correspondence should be addressed to Quanxin Zhu; zqx22@126.com and Jialin Huang; jialinh2880@163.com
}

Received 24 September 2020; Revised 13 March 2021; Accepted 29 March 2021; Published 13 April 2021

Academic Editor: Eulalia Mart nez

Copyright (C) 2021 Ruofeng Rao et al. This is an open access article distributed under the Creative Commons Attribution License, which permits unrestricted use, distribution, and reproduction in any medium, provided the original work is properly cited.

\begin{abstract}
In this study, the authors utilize mountain pass lemma, variational methods, regularization technique, and the Lyapunov function method to derive the unique existence of the positive classical stationary solution of a single-species ecosystem. Particularly, the geometric characteristic of saddle point in the mountain pass lemma guarantees that the equilibrium point is the ground state stationary solution of the ecosystem. Based on the obtained uniqueness result, the authors use the Lyapunov function method to derive the globally exponential stability criterion, which illuminates that under some suitable conditions, a certain internal competition is conducive to the global stability of the population, and a certain amount of family planning is conducive to the overall stability of the population. Most notably, the regularity technique of weak stationary solution employed in this study can also be applied to some existing literature related with time-delays reaction-diffusion systems for the purpose of regularization of weak solutions. Finally, an illuminative numerical example shows the effectiveness of the proposed methods.
\end{abstract}

\section{Introduction}

The logistic system is one of the most classical models in ecology and mathematics, which is very important to the development of ecology [1]. It is usually expressed as

$$
\frac{\mathrm{d} x}{\mathrm{~d} t}=r x(t)\left(1-\frac{x(t)}{K}\right)
$$

where $x(t)$ represents the density or quantity of population $x$ at time $t$, and $r>0$ and $K$ represent the intrinsic growth rate of population and environmental capacity, respectively. In 2011, Xiaoling Zou and Ke Wang investigated the long time behavior of the following stochastic ecosystem for single species ([2], Theorem 2 ):

$$
\mathrm{d} x=x[a-b x] \mathrm{d} t+\alpha x \mathrm{~d} B(t) .
$$

where $a>0$ and $b>0$ describe the growth rate and the intraspecific competition; $\alpha>0$ measures the intensity of the environmental disturbances. In recent years, model (2) has been widely adopted in many applications (e.g., [3-5]). A large number of facts have shown that the spatial scale and structure of the environment can affect population interaction [6,7] and community composition [8]. In the landmark document [9], Kellam gave a large number of observations, which had a profound impact on the study of spatial ecology. First, he linked random walk with diffusion equation. The former is a description of the individual movement of some theoretical biological species, and the latter is a description of the density distribution of biological populations. He uses the data of muskrat transmission in Central Europe to prove that this connection is reasonable for small animals. Second, he combines diffusion with 
population dynamics and effectively introduces the reactiondiffusion equation into theoretical ecology.

In recent years, many dynamical systems, including reaction systems, have been considered as the theoretical branches of dynamical systems $[10,11,12]$. In addition, the competition within the population is the participation of the adult population, and there is a period from infancy to adulthood. At the same time, this time-delay problem is affected by many stochastic factors such as weather, temperature, humidity, and so on. Besides, in real life, the factors that affect population growth do not change only at a fixed time but also occur randomly. When these factors occur, the system will also change randomly. As is well known, the phenomenon of population clustering is widespread in nature, which is likely to be completely affected by environmental factors and human factors. In this case, the growth curve of mosquitoes or small fish will be different from the previous form. This phenomenon can be expressed as a switch between two environmental states because the switching between different environments is not memory free; therefore, one can use continuous time Markov chain to model the situation of environment switching $[13,14,15]$.

Inspired by some ideas and methods of related literature $[16-31]$, particularly $[17,18]$, we are to investigate the stability of stationary density of a single-species model with diffusion and delayed feedback under natural state. This study has the following highlights:

(i) As far as we know, it is the first study to investigate the stability of stationary density of a single-species model with diffusion and delayed feedback under the Dirichlet zero boundary value. And the Dirichlet boundary value can well simulate the fact that the species lives in its biosphere, while the population density tends to zero at the boundary of biosphere due to the harsh condition. Different from exiting literature involved in Neumann zero boundary value which implies that there is no animal migration at the edge of the biosphere, the Dirichlet boundary value of this study admits the fact of animal migration, but no animals under study live on the border for a long time.

(ii) It is the first comprehensive application of mountain pass lemma, variational technique, and the Lyapunov function method to derive the unique existence of globally exponentially stable positive stationary solution of a single-species model with diffusion and delayed feedback under the Dirichlet zero boundary value.

(iii) The obtained stability criterion illuminates that under some suitable conditions, a certain internal competition is conducive to the overall stability of the population, and a certain amount of family planning is conducive to the overall stability of the population.

(iv) Different from many existing literature related with the global stability of discontinuous systems [30-32] and time-delays reaction-diffusion systems [33, 34], the weak stationary solution is regularized in this study. Most notably, the regularity technique of weak stationary solutions can also be applied to such existing literature $[24,25,33,34]$ for the purpose of regularization of weak solutions.

In next sections, the authors have made the following arrangement:

In Section 2, the authors present some descriptions on the ecosystem, and some necessary definitions and lemmas are presented. In Section 3, the authors utilize the existence technique and regularity method employed in [17] to derive the existence of positive strong stationary solution of the ecosystem. Moreover, utilizing the uniqueness technique used in $[18,35]$ results in the unique existence of the stationary solution. Finally, the Lyapunov function method is applied to derive the stability criterion. In Section 4, numerical example and comparisons are given. And in final section, conclusions and further considerations are proposed.

For convenience, throughout of this study, we denote by $\lambda_{1}$ the first positive eigenvalue of Laplace operator $-\Delta$ in $H_{0}^{1}(\Omega)$. Denote $u^{+}=\max \{u, 0\}, u^{-}=\min \{u, 0\}$. Denote by $\|u\|=\sqrt{\int_{\Omega}|\nabla u(x)|^{2} \mathrm{~d} x}$, the norm of $H_{0}^{1}(\Omega)$, and by $\lambda_{1}$, the first positive eigenvalue of Laplace operator $-\Delta$ in $H_{0}^{1}(\Omega)$. Besides, we denote $|v|=\left(\left|v_{1}\right|,\left|v_{2}\right|\right)^{T}$ for $v=\left(v_{1}, v_{2}\right)^{T} \in \mathbb{R}^{2}$ and for matrix $C=\left(c_{i j}\right)_{2 \times 2}$.

\section{System Descriptions}

Denote by $(\Upsilon, \mathscr{F}, \mathbb{P})$ the complete probability space with a natural filtration $\left\{\mathscr{F}_{t}\right\}_{t>0}$. Let $S=\left\{1,2, \ldots, n_{0}\right\}$ and the random form process $\{r(t):[0,+\infty) \longrightarrow S\}$ be a homogeneous, finite-state Markovian process with right continuous trajectories with generator $\Pi=\left(\gamma_{i j}\right)_{n_{0} \times n_{0}}$ and transition probability from mode $i$ at time $t$ to mode $j$ at time $t+\delta, i, j \in S$,

$$
\mathbb{P}(r(t+\delta)=j \mid r(t)=i)= \begin{cases}\gamma_{i j} \delta+o(\delta), & j \neq i, \\ 1+\gamma_{i j} \delta+o(\delta), & j=i,\end{cases}
$$

where $\gamma_{i j} \geq 0$ is the transition probability rate from $i$ to $j(j \neq i) \quad$ and $\quad \gamma_{i i}=-\sum_{j=1, j \neq i}^{n_{0}} \gamma_{i j}, \quad \delta>0 \quad$ and $\lim \backslash$ limits_ $\delta \longrightarrow 0 o(\delta) / \delta=0$.

Consider the following ecosystem with diffusion and delayed feedback,

$$
\begin{cases}\frac{\partial u(t, x)}{\partial t}=q \Delta u(t, x)+u(t, x)[a-b u(t, x)]+c(r(t))[u(t, x)-u(t-\tau(t), x)]+\Lambda(u), & t \geq 0, x \in \Omega, \\ u(t, x)=0, & x \in \partial \Omega, t \geq 0,\end{cases}
$$


where $\Lambda(u)$ is the external input, $a>0$ and $b>0$ describe the growth rate and the intraspecific competition, and $\Omega \in R^{3}$ is the bounded domain with its boundary $\partial \Omega$ and is also a $C^{2, \sigma}$ domain in $R^{3}$ (e.g., [17]). It is also suitable to the case that the species lives in two dimensional plane ([35], Remark 2.1).

Remark 1. Because only the adult is competitive for survival and there is a mature period from the larva to the adult, we consider the time-delayed system in this study, which is better to simulate this maturity problem. Particularly, the gain coefficient of time-delay feedback can be derived from the statistical data of the observed system.

Throughout this study, we assume (H1) the positive function $\Lambda$ is only a microperturbation. That is, there exists a positive number $\varepsilon>0$ small enough such that

$$
\lim _{u \longrightarrow \infty} \frac{\Lambda(u)}{u^{\theta}}=\varepsilon=\lim _{u \longrightarrow \infty} \frac{\Lambda(u)}{u},
$$

where $2^{*}-1>\theta=\left(\theta_{2} / \theta_{1}\right)>2$ with $\theta_{2}$ and $\theta_{1}$ being a pair of coprime odd numbers. And $\Lambda(\cdot)$ is continuous and $\Lambda(u) \geq 0$ for all $u \geq 0$. Here, $2^{*}$ is the Sobolev critical exponent. In addition, $\Lambda(u)=0$ for all $u \leqslant 0$.

Remark 2. In essence, the restrictive condition $\theta=\left(\theta_{2} / \theta_{1}\right)>2$ with $\theta_{2}$ and $\theta_{1}$ is set to ensure that the function $u^{\theta}$ is odd, so that its primitive function is even. Thereby, we can also assume in $\mathrm{H} 1$ that the function $u^{\theta}$ is odd.

Let $u_{*}(x)$ be a stationary solution of system (4) implies that $u_{*}(x)$ is a solution of the following equation:

$$
-q \Delta u=a u-b u^{2}+\Lambda(u), \quad x \in \Omega ;\left.u\right|_{\partial \Omega}=0 .
$$

Of course, each solution of equation (6) must be one of the solutions of system (4).

Definition 1. The stationary solution $u_{*}(x)$ of system (4) is called the ground-state stationary solution of system (4) if $u_{*}(x)$ is the ground-state solution of equation (6).

Definition 2. A solution $u_{*}(x)$ of equation (6) is called the strong solution of equation (6) if $u_{*}(x) \in C^{2}(\Omega)$.

To prove the main result of this study, we need the following lemmas (e.g., $[17,20])$ :

Lemma 1. Consider the following equation:

$$
\left\{\begin{array}{l}
-\Delta u=f(x, u), \quad x \in \Omega \\
\left.u\right|_{\partial \Omega}=0
\end{array}\right.
$$

where $\Omega$ is a $C^{k+2, \alpha}$ domain of $R^{n}$, and $f$ satisfies the following conditions:

(a) There exists $0<r \leq 1$ such that for any given positive number $M$,

$$
f(x, u) \in C^{k, r}\left(\bar{\Omega} \times[-M, M], R^{1}\right),
$$

(b) If $n \geq 3$ and $s=2^{*}-1$, or $n \leq 2$ and $s>1$, then $f(x, u)=O\left(|u|^{s}\right)($ as $|u| \longrightarrow \infty)$ holds uniformly for $x \in \bar{\Omega}$

(c) $\lim _{u \longrightarrow 0}(f(x, u) / u)=a(x) \in L^{\infty}(\Omega)$.

Then, the solution of equation (6) in $H_{0}^{1}(\Omega)$ is the strong solution. In addition, $u \in C^{k+2, \delta}$ for $\delta=\alpha r^{k+1}$.

Lemma 2. Let $u \in H_{0}^{1}(\Omega)$. Then, there is a conclusion that $u^{+}, u^{-},|u| \in H_{0}^{1}(\Omega)$. Besides, $\nabla u^{+}=\nabla u$ if $u>0, \nabla u^{+}=0$ if $u \leqslant 0, \nabla u^{-}=0$ if $u \geq 0$, and $\nabla u^{-}=\nabla u$ if $u<0$. In addition, $\nabla|u|=\nabla u$ if $u>0$ and $\nabla|u|=-\nabla u$ if $u<0$. Besides, $\nabla|u|=0$ if $u=0$.

Lemma 3 (Mountain pass lemma without the PS condition). Let $X$ be a Banach space, $\Psi \in C^{1}(X, \mathbb{R})$, satisfying $\Psi(0)=0$, and there exists $\rho>0$ such that $\left.\Psi\right|_{\partial B_{\rho}(0)} \geq \alpha>0$. Besides, there is $e \in X \backslash \overline{B_{\rho}(0)}$, such that $\Psi(e) \leq 0$. Let $\Gamma$ be the set of all paths connecting 0 and $e$, that is,

$$
\Gamma=\left\{\psi \in C\left([0,1], H_{0}^{1}(\Omega)\right): \psi(0)=0, \psi(1)=e\right\} .
$$

Set

$$
c_{*}=\inf _{\psi \in \Gamma} \max _{s \in[0,1]} \Psi(\psi(s)) .
$$

Then, $c_{*} \geq \alpha$, and $\Psi$ possesses a critical sequence on $c_{*}$.

Remark 3. Lemma 3 is the mountain pass lemma without the PS condition (e.g., [17]). If, in addition, $\Psi$ satisfies the PS condition, then $c_{*}$ is a critical value of $\Psi$. Besides, let $\Psi$ be the functional corresponding to equation (6), then $u_{*}(x)$ must be a ground state solution of equation (6) if $u_{*}(x)$ is a critical point of the functional $\Psi$ with $\Psi\left(u_{*}(x)\right)=c_{*}$ defined in (10) of Lemma 3.

\section{Main Result}

First, we may present the existence of a stationary strong solution $u_{*}(x)$ of system 2.1. In addition, it is necessary to guarantee that $u_{*}(x) \geq 0$ and $u_{*}(x) \neq 0$, which may be proved as follows:

Theorem 1. Suppose the condition H1 holds, and if

$$
a<q \lambda_{1} .
$$

Then, there is a ground-state strong stationary solution for system (4).

Proof. Let $u_{*}(x)$ be a positive stationary solution of system (4), satisfying

$$
-q \Delta u=a u-b u^{2}+\Lambda(u), \quad u(x)>0, x \in \Omega ;\left.u\right|_{\partial \Omega}=0,
$$

whose functional is 


$$
\Psi(u)=\int_{\Omega} \frac{|\nabla u|^{2}+\mu u^{2}-\mu\left(u^{+}\right)^{2}}{2} \mathrm{~d} x-\frac{a}{2 q} \int_{\Omega}\left(u^{+}\right)^{2} \mathrm{~d} x+\frac{b}{3 q} \int_{\Omega}\left(u^{+}\right)^{3} \mathrm{~d} x-\int_{\Omega} \tilde{\Lambda}\left(u^{+}\right) \mathrm{d} x,
$$

where $\mu>0$ is a constant, and $\widetilde{\Lambda}(u)=\int_{0}^{u} \Lambda(s) \mathrm{d} s$. It is obvious that $\Psi \in C^{1}\left(H_{0}^{1}(\Omega), R^{1}\right)$, and a critical point of the functional $\Psi$ is corresponding to the solution of equation (12). Next, we claim that $\Psi$ satisfies the condition of the mountain road geometry. In fact, obviously $\Psi(0)=0$.

The microperturbation condition $(\mathrm{H} 1)$ yields that there are there positive constants $c_{0}, m_{1}, m_{2}$ with $m_{1}<1<m_{2}$ such that

$$
\varepsilon-\frac{1}{2} \varepsilon<\left|\frac{\Lambda}{u^{\theta}}\right|<\varepsilon+\frac{1}{2} \varepsilon, \quad u \in\left(m_{2},+\infty\right)
$$

or

$$
\begin{aligned}
\frac{1}{2} \varepsilon u & \leq \Lambda(u) \leq \frac{3}{2} \varepsilon u, \quad u \in\left[0, m_{1}\right) \\
\frac{1}{2} \varepsilon u^{\theta} & \leq \Lambda(u) \leq \frac{3}{2} \varepsilon u^{\theta}, \quad u \in\left(m_{2},+\infty\right) ; \\
0 & \leq \Lambda(u) \leq c_{0}, \quad u \in\left[m_{1}, m_{2}\right]
\end{aligned}
$$

which implies

$$
\begin{aligned}
& \Lambda(u) \leq \frac{3}{2} \varepsilon u, \quad u \in\left[0, m_{1}\right) \\
& \Lambda(u) \leq \frac{3}{2} \varepsilon u^{\theta}, \quad u \in\left(m_{2},+\infty\right) \\
& \Lambda(u) \leq c_{0}, \quad u \in\left[m_{1}, m_{2}\right]
\end{aligned}
$$

and then,

$$
\Lambda(u) \leq \frac{3}{2} \varepsilon u+\frac{3}{2} \varepsilon u^{\theta}+c_{0} \leq \frac{3}{2} \varepsilon u+\frac{3}{2} \varepsilon u^{\theta}+c_{0} \frac{u^{\theta}}{m_{1}^{\theta}}, \quad \forall u \geq 0 .
$$

Moreover,

$$
\tilde{\Lambda}(u)=\int_{0}^{u} \Lambda(s) \mathrm{d} s \leq \frac{3}{4} \varepsilon u^{2}+\left(\frac{3}{2} \varepsilon+\frac{c_{0}}{m_{1}^{\theta}}\right) \frac{1}{1+\theta} u^{1+\theta}, \quad \forall u \geq 0 .
$$

Next, (13) and (18), Poincare inequality and Sobolev embedding theorem yield that there is a positive constant $c_{1}>0$ such that

$$
\Psi(u) \geq \frac{1}{2} \int_{\Omega}|\nabla u|^{2} \mathrm{~d} x-\frac{a}{2 q} \int_{\Omega} u^{2} \mathrm{~d} x-\int_{\Omega} \widetilde{\Lambda}\left(u^{+}\right) \mathrm{d} x \geq\left(\frac{1}{2}-\frac{a}{2 q \lambda_{1}}-\frac{3}{4} \varepsilon\right)\|u\|^{2}-c_{1}\|u\|^{1+\theta}
$$

Besides, (11) and small $\varepsilon>0$ lead to

$$
\frac{1}{2}-\frac{a}{2 q \lambda_{1}}-\frac{3}{4} \varepsilon>0,
$$

which together with $\theta>2$ means that we can set

$$
\begin{aligned}
& \rho=\left(\frac{1}{2 c_{1}}\left(\frac{1}{2}-\frac{a}{2 q \lambda_{1}}-\frac{3}{4} \varepsilon\right)\right)^{(1 /(\theta-1))}, \\
& \alpha=\frac{1}{2}\left(\frac{1}{2}-\frac{a}{2 q \lambda_{1}}-\frac{3}{4} \varepsilon\right)\left(\frac{1}{2 c_{1}}\left(\frac{1}{2}-\frac{a}{2 q \lambda_{1}}-\frac{3}{4} \varepsilon\right)\right)^{(2 /(\theta-1))},
\end{aligned}
$$

such that $\left.\Psi\right|_{\partial B_{\rho}(0)} \geq \alpha>0$.

On the other hand, it follows by (15) and $m_{1}<1$ that

$$
\begin{aligned}
& \frac{1}{2} \varepsilon u^{\theta} \leq \frac{1}{2} \varepsilon u \leq \Lambda(u), \quad u \in\left[0, m_{1}\right) \\
& \frac{1}{2} \varepsilon u^{\theta} \leq \Lambda(u), \quad u \in\left(m_{2},+\infty\right) \\
& \Lambda(u) \geq 0, \quad u \in\left[m_{1}, m_{2}\right]
\end{aligned}
$$

which implies

$$
\Lambda(u) \geq \frac{1}{2} \varepsilon u^{\theta}, \quad \forall u \geq 0,
$$

or

$$
\widetilde{\Lambda}(u) \geq \frac{1}{2(1+\theta)} \varepsilon u^{1+\theta}, \quad \forall u \geq 0 .
$$

We may select $u \in H_{0}^{1}(\Omega)$ with $u \geq 0$, and then,

$$
\begin{aligned}
\Psi(u) & =\frac{1}{2} \int_{\Omega}|\nabla u|^{2} \mathrm{~d} x-\frac{a}{2 q} \int_{\Omega} u^{2} \mathrm{~d} x+\frac{b}{3 q} \int_{\Omega} u^{3} \mathrm{~d} x \mathrm{~d} x-\int_{\Omega} \tilde{\Lambda}(u) \mathrm{d} x \\
& \leq \frac{1}{2} \int_{\Omega}|\nabla u|^{2} \mathrm{~d} x-\frac{a}{2 q} \int_{\Omega} u^{2} \mathrm{~d} x+\frac{b}{3 q} \int_{\Omega} u^{3} \mathrm{~d} x \mathrm{~d} x-\int_{\Omega} \frac{1}{2(1+\theta)} \varepsilon u^{1+\theta} \mathrm{d} x .
\end{aligned}
$$


Let $\varphi_{1}(x)>0$ with $\|\varphi\|=1$ be the eigenfunction corresponding to the first positive eigenvalue $\lambda_{1}$ (e.g., [18]), and set $u=s \varphi$; then, $\Psi(s \varphi) \longrightarrow-\infty$ if $s \longrightarrow+\infty$, so that there exists $s_{0}>0$ satisfying $\left\|s_{0} \varphi\right\| \geq \rho$ and $\Psi(s \varphi)<0$. And then, $\Psi$ satisfies the condition of the mountain road geometry. According to mountain pass lemma, let $\Gamma$ be the set of all paths connecting 0 and $e=s_{0} \varphi$. That is,

$$
\Gamma=\left\{\psi \in C\left([0,1], H_{0}^{1}(\Omega)\right): \psi(0)=0, \psi(1)=e\right\} .
$$

Set

$$
c_{*}=\inf _{\psi \in \Gamma} \max _{s \in[0,1]} \Psi(\psi(s)) .
$$

Then, $c_{*} \geq \alpha$, and $\Psi$ possesses a critical sequence on $c_{*}$, say $\left\{u_{k}\right\} \subset H_{0}^{1}(\Omega)$ with $\Psi\left(u_{k}\right) \longrightarrow c_{*}$ and $\Psi^{\prime}\left(u_{k}\right) \longrightarrow 0$ in $\left(H_{0}^{1}(\Omega)\right)^{*}$. That is, for any given $\epsilon>0$, there exists $k$ big enough such that

$$
\int_{\Omega} \frac{\left|\nabla u_{k}\right|^{2}+\mu u_{k}^{2}-\mu\left(u_{k}^{+}\right)^{2}}{2} \mathrm{~d} x-\frac{a}{2 q} \int_{\Omega}\left(u_{k}^{+}\right)^{2} \mathrm{~d} x+\frac{b}{3 q} \int_{\Omega}\left(u_{k}^{+}\right)^{3} \mathrm{~d} x-\int_{\Omega} \tilde{\Lambda}\left(u_{k}^{+}\right) \mathrm{d} x=\Psi\left(u_{k}\right)=c_{*}+o(1)
$$

and

$$
\int_{\Omega}\left(\left|\nabla u_{k}\right|^{2}+\mu u_{k}^{2}-\mu u_{k}^{+} u_{k}-\frac{a}{q} u_{k}^{+} u_{k}+\frac{b}{q}\left(u_{k}^{+}\right)^{2} u_{k}-\Lambda\left(u_{k}^{+}\right) u_{k}\right) \mathrm{d} x=\left\langle\Psi^{\prime}\left(u_{k}\right), u_{k}\right\rangle
$$

$$
\left\langle\Psi^{\prime}\left(u_{k}\right), u_{k}\right\rangle \leq \varepsilon\left\|u_{k}\right\|,
$$

where $o(1)$ represents such an infinitesimal that $o(1) \longrightarrow 0$ when $k \longrightarrow \infty$.

(17) yields

$$
\Lambda(u) \leq \frac{3}{2} \varepsilon u+\frac{3}{2} \varepsilon u^{\theta}+c_{0} .
$$

Similar as the methods of [17], (24)-(27), employing (29)-(33) results in

$$
\left(\frac{1}{2}-\frac{1}{1+\theta}\right)\left(1-\frac{a}{\lambda_{1} q}-c_{3} \varepsilon\right)\left\|u_{k}\right\|^{2}+c_{4} \int_{\Omega}\left(u^{+}\right)^{3} \mathrm{~d} x \leq c_{*}+o(1)+\frac{\varepsilon}{1+\theta}\left\|u_{k}\right\|
$$

where $c_{3}, c_{4}$ both are the positive constants, and $1-\left(a / \lambda_{1} q\right)-c_{3} \varepsilon>0$ due to the small $\varepsilon$, which means the boundedness of $\left\{u_{k}\right\}$. Due to $\theta<2^{*}-1$, equation (12) is the subcritical growth. It is a routine proof of the fact that $\left\{u_{k}\right\}$ is sequently compact, say $u_{k} \longrightarrow u_{*}(x)$ in $H_{0}^{1}(\Omega)$ and $\Psi\left(u_{*}(x)\right)=c_{*} \geq \alpha>0$, which implies $u_{*}(x) \neq 0$. Besides, $u_{*}(x)$ is the critical point of $\Psi$, so that

$$
\int_{\Omega}\left(\nabla u_{*}(x) \nabla \vartheta+\mu u_{*}(x) \vartheta-\mu u_{*}(x)^{+} \vartheta-\frac{a}{q} u_{*}(x)^{+} \vartheta+\frac{b}{q}\left(u_{*}(x)^{+}\right)^{2} \vartheta-\Lambda\left(u_{*}(x)^{+} \vartheta\right)\right) \mathrm{d} x=0 .
$$

In (35), setting $\vartheta=u_{*}(x)^{-}$, Lemma 2 leads to

$$
\mu \int_{\Omega}\left|u_{*}(x)^{-}\right|^{2} \mathrm{~d} x \leq \int_{\Omega}\left(\left|\nabla u_{*}(x)^{-}\right|^{2}+\mu\left|u_{*}(x)^{-}\right|^{2}\right) \mathrm{d} x=0,
$$

which implies that $u_{*}(x)^{-}=0$ a.e. $x \in \Omega$. Now, we have proved that $u_{*}(x) \geq 0$ and $u_{*}(x) \neq 0$.

Similar as that of [17], now we claim that the abovementioned $u_{*}(x)$ is the strong solution.

Indeed, $u_{*}(x) \neq 0$ is the nonnegative solution of the following Dirichlet problem:

$$
\left\{\begin{array}{l}
-\Delta u=f(x, u), \quad x \in \Omega \\
\left.u\right|_{\partial \Omega}=0
\end{array}\right.
$$

where

$$
f(x, u)=\frac{1}{q}\left[a u-b u^{2}+\Lambda(u)\right]
$$

It is easy from the assumptions on $\Lambda$ to verify that $f$ satisfies the conditions (a)-(c); then, Lemma 1 yields that $u_{*}(x)$ is the strong solution. 
Set $v(t, x)=u(t, x)-u_{*}(x)$. Since $u_{*}(x)$ is a stationary solution of system (4), system (4) is equivalent to the following system:

$$
\begin{cases}\frac{\partial v(t, x)}{\partial t}=q \Delta v(t, x)+\left(a+c_{r}-2 b u_{*}(x)\right) v(t, x)+g(v(t, x))-b v^{2}(t, x)-c_{r} v(t-\tau(t), x), & t \geq 0, x \in \Omega \\ v(t, x)=0, & x \in \Omega, t \geq 0\end{cases}
$$

where

$$
g(v(t, x))=\Lambda(u(t, x))-\Lambda\left(u_{*}(x)\right) .
$$

Obviously, $u_{*}(x)$ of system (4) is corresponding to the zero solution of system (38).

Equip system (38) with the initial value,

$$
v(s, x)=\phi(s, x),(s, x) \in[-\tau, 0] \times \Omega .
$$

Moreover, we give some suitable assumptions as follows: $\mathrm{H} 2$. There are positive numbers $M_{0}, N_{0}$, such that

$$
0<N_{0} \leq u \leq M_{0} .
$$

H3. There is a positive number $M_{1}>0$, such that

$$
\left|\Lambda\left(s_{1}\right)-\Lambda\left(s_{2}\right)\right| \leq M_{1}\left|s_{1}-s_{2}\right|, \quad \forall s_{1}, s_{2} \in R^{1} .
$$

Remark 4. Everyone knows the fact that the population density of any species must have the bounded below or the species will die out. For example, when the population density of whales is lower than a certain degree, it will be difficult for male and female whales to meet each other in the vast sea, leading to the extinction of the species. Besides, due to the limited resource, the population density of any species

must have supper boundedness. So, the condition $\mathrm{H} 2$ is a suitable assumption.

There are some techniques on the existence and uniqueness of positive stationary solution in the proofs ([18], Theorems 1-2), which are also employed in the proofs of ([35], Theorems 1-2). But the methods used in the proof of Theorem 1 in this study are different from those of both $[18,35]$. Besides, we are willing to consider similarly the uniqueness of the positive stationary solution in this study.

Theorem 2. If all the conditions of Theorem 1 hold, and if, in addition,

$$
\lambda_{1} q>a-2 b N_{0}+M_{1}
$$

then $u_{*}(x)$ is the unique stationary solution of system (11).

Proof. Let $u, w$ both are the stationary solutions of system (4). First, the condition $\mathrm{H} 2$ yields

$$
\begin{gathered}
(u-w)\left[-b\left(u^{2}-w^{2}\right)\right]=-b(u-w)^{2}(u+w) \leq-2 b N_{0}(u-w)^{2}, \\
(u-w)(\Lambda(u)-\Lambda(w)) \leq|u-w \| \Lambda(u)|-\Lambda(w) \leq M_{1}(u-w)^{2} .
\end{gathered}
$$

Since $u, w$ both are the stationary solutions of system (4), then Poincare inequality yields

$$
\lambda_{1} q\|u-w\|_{L^{2}(\Omega)}^{2} \leq \int_{\Omega}(u-w)\left[a(u-w)-b\left(u^{2}-w^{2}\right)+\Lambda(u)-\Lambda(w)\right] \mathrm{d} x \leq \int_{\Omega}\left[a-2 b N_{0}+M_{1}\right](u-w)^{2} \mathrm{~d} x
$$

which proves $u=w$, and the proof is completed.
Theorem 3. Suppose the conditions H1-H3 and (45) hold and if there are positive numbers $p_{r}(r \in S), k_{1}>0$ such that

$$
\min _{r \in S}\left(2 \lambda_{1} q+2\left(2 b N_{0}-a-c_{r}\right)-2 M_{1}-2 b\left(M_{0}-N_{0}\right)-c_{r} k_{1}-\frac{1}{p_{r}} \sum_{j \in S} \gamma_{r j} p_{j}\right)>\max _{r \in S}\left(c_{r} k_{1}^{-1}\right) \geq 0
$$

then the null solution of system (38) with the initial value (40) is the globally exponential input-to-state stability; at the same time, $u_{*}(x)$ is the globally exponential input-to-state stability at the convergence rate $(\lambda / 2)$, where $\alpha=\min _{r \in S}\left(2 \lambda_{1} q+2\left(2 b N_{0}-a-c_{r}\right)-\right.$ $\left.2 M_{1}-2 b \quad\left(M_{0}-N_{0}\right) \quad-c_{r} k_{1} \quad-\left(1 / p_{r}\right) \sum_{j \in S} \gamma_{r j} p_{j}\right)$, $\beta=\max _{r \in S}\left(c_{r} k_{1}^{-1}\right)$, and $\lambda$ is the unique positive solution of $\lambda=\alpha-\beta e^{\lambda \tau}$. 
Proof. Consider the following Lyapunov function:

$$
V(t, x, v, r)=\int_{\Omega} p_{r} v^{2}(t, x) \mathrm{d} x,
$$

where $p_{r}$ is a positive number for each $r$.

First, $\mathrm{H} 2$ yields

$$
v=u-u_{*}(x) \geq N_{0}-M_{0} \Rightarrow v^{3} \geq\left(N_{0}-M_{0}\right) v^{2} .
$$

$\mathrm{H} 3$ yields

$$
|v g(v)| \leq|v| M_{1}|v|=M_{1} v^{2} .
$$

Let $\mathscr{L}$ be the weak infinitesimal operator (e.g., [21]), such that

$$
\begin{aligned}
\mathscr{L} V & \leq \int_{\Omega}\left(-2 \lambda_{1} q p_{r} v^{2}+2 p_{r}\left(a+c_{r}-2 b u_{*}(x)\right) v^{2}+2 p_{r} v g(v(t, x))-2 b p_{r} v^{3}-2 p_{r} c_{r} v v(t-\tau(t), x)+\sum_{j \in S} \gamma_{r j} p_{j}\right) \mathrm{d} x \\
& \leq-\left(2 \lambda_{1} q+2\left(2 b N_{0}-a-c_{r}\right)-2 M_{1}-2 b\left(M_{0}-N_{0}\right)-c_{r} k_{1}-\frac{1}{p_{r}} \sum_{j \in S} \gamma_{r j} p_{j}\right) V+c_{r} k_{1}^{-1} V_{\tau} \\
& \leq-\left[\min _{r \in S}\left(2 \lambda_{1} q+2\left(2 b N_{0}-a-c_{r}\right)-2 M_{1}-2 b\left(M_{0}-N_{0}\right)-c_{r} k_{1}-\frac{1}{p_{r}} \sum_{j \in S} \gamma_{r j} p_{j}\right)\right] V+\max _{r \in S}\left(c_{r} k_{1}^{-1}\right) V_{\tau} \mathrm{ed},
\end{aligned}
$$

where $V_{\tau}(t)=\sup _{t-\tau \leq s \leq t} V(s)$.

Now, ([36], Lemma 2) yields that

$$
\min _{r \in S} p_{r}\|v\|_{L^{2}(\Omega)}^{2} \leq V(t, r) \leq \max _{r \in S} p_{r}\|v\|_{\tau}^{2} e^{-\lambda \tau\left(t-t_{0}\right)}, \quad t \geq 0,
$$

where $t_{0}=0,\|v(t)\|_{\tau}^{2}=\sup _{t-\tau \leq s \leq t}\|v(s)\|_{L^{2}(\Omega)}^{2}$. This completes the proof.

Remark 5. In this study, we employ mountain pass lemma and variational technique to derive the existence of positive stationary solution, which is different from the methods in [18]. Particularly, ground-state solution is more suitable to practical engineering (e.g., [36-40]). Besides, the equilibrium points of ecosystems with the Neumann boundary value are always constants solutions, while equilibrium points of the system (4) are always the nontrivial solutions of the corresponding elliptic equation, which need the existence criterion of the solutions for the elliptic equation. And it adds the computation complexity of the results obtained in this study. Also, our model and method are different from those in [41-46].

\section{Numerical Example}

Example 1. In system (4), let $\Omega=(-(1 / 2),(1 / 2)) \times(-(1 / 2),(1 / 2)) \times(-(1 / 2),(1 / 2))$, then $\lambda_{1} \geq 3$ ([18], Remark 14). $q=0.1, a=0.2$, then $a<q \lambda_{1}$, and condition (11) holds. Set

$$
\Lambda(u)=\left\{0, \quad u \in(-\infty, 0] \varepsilon u, u \in[0,1] \varepsilon u^{(7 / 3)}, \quad u \in[1,+\infty)\right.
$$

where $\varepsilon=0.00001$, then $\mathrm{H} 1$ holds. Let $b=0.1, N_{0}=2, M_{0}=10$, then direct computation yields $M_{1}=0.003$, and obviously, (45) holds. Then, Theorems 1-2 yield $u_{*}(x)$ is the unique stationary solution of system (4), and $u_{*}(x)$ is the ground state stationary strong solution of system (4).

Moreover, set $\tau=0.5, S=\{1,2\}$, and $c_{1}=0.01, c_{2}=0.02$, $\gamma_{11}=-0.01, \gamma_{12}=0.01 ; \gamma_{21}=-0.02, \gamma_{22}=0.02, p_{1}=$ $0.9999, p_{2}=1.0001, k_{1}=1$, and direct computation yields (46) holds for $r=1,2$, and $\lambda=0.4769$. According to Theorem 3 , the null solution of system (38) with the initial value (40) is globally exponential input-to-state stability; at the same time, $u_{*}(x)$ is globally exponential input-to-state stability at the convergence rate $23.85 \%$.

In Example 1, replacing $\varepsilon=0.00001$ with $\varepsilon=0.0001$ and other data unchanged, direct computation yields the convergence rate $(\lambda / 2)=21.59 \%$.

Remark 6. Table 1 provides that under some suitable conditions, the smaller the external input disturbance, the faster the stability of the natural ecosystem (Figure 1).

In example 1 , replacing $b=0.1$ with $b=0.2$ and other data unchanged, direct computation yields the convergence rate $(\lambda / 2)=28.98 \%$.

Remark 7. Table 2 provides that a certain degree of inhibition and competition within the population is beneficial to the overall stability of the population for the natural ecosystem of a single-species model. Figure 2 shows that the bigger the intrapopulation competition intensities, the faster the stability of a single-species system.

In Example 1, replacing $a=0.2$ with $a=0.23$ and other data unchanged, direct computation yields the convergence rate $(\lambda / 2)=19.08 \%$. 
TABLE 1: Comparisons the influences on the convergence rate $(\lambda / 2)$ under different perturbations with the same other data.

\begin{tabular}{lcc}
\hline & $\varepsilon=0.00001$ & $\varepsilon=0.0001$ \\
\hline Interference degree & Smaller & Bigger \\
Convergence rate $(\lambda / 2)$ & $23.85 \%$ & $21.59 \%$ \\
\hline
\end{tabular}

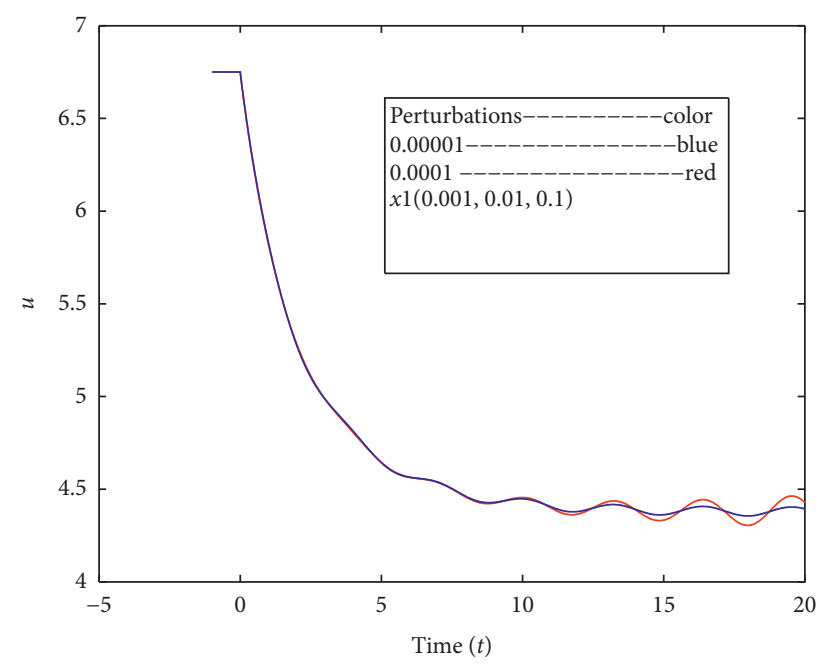

Figure 1: Sectional curves of $u(t, x)$ with $\varepsilon=0.00001$ (blue) and $\varepsilon=0.0001$ (red) at $x_{1}$.

TABLE 2: Comparisons of the influences on the convergence rate $(\lambda / 2)$ under different intrapopulation competition intensities with the same other data.

\begin{tabular}{llc}
\hline & $b=0.1$ & $b=0.2$ \\
\hline Intraspecific competition & Smaller & Bigger \\
Convergence rate $(\lambda / 2)$ & $23.85 \%$ & $28.98 \%$ \\
\hline
\end{tabular}

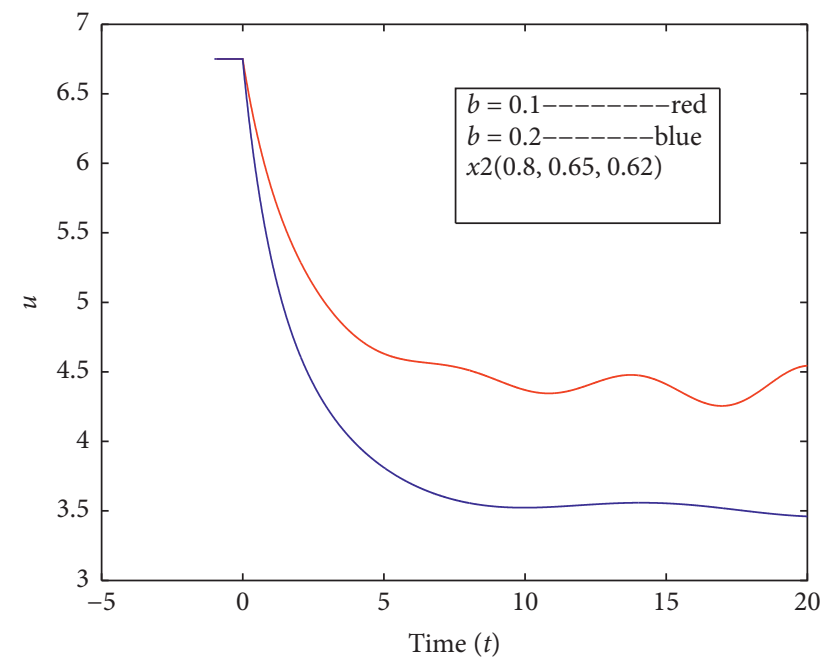

FIGURE 2: Sectional curves of $u(t, x)$ with $b=0.1$ (red) and $b=0.2$ (blue) at $x_{2}$. 
TABLE 3: Comparisons the influences on the convergence rate $(\lambda / 2)$ under different growth rates with the same other data.

\begin{tabular}{llc}
\hline & $a=0.2$ & $a=0.23$ \\
\hline Growth rates & Smaller & Bigger \\
Convergence rate $(\lambda / 2)$ & $23.85 \%$ & $19.08 \%$ \\
\hline
\end{tabular}

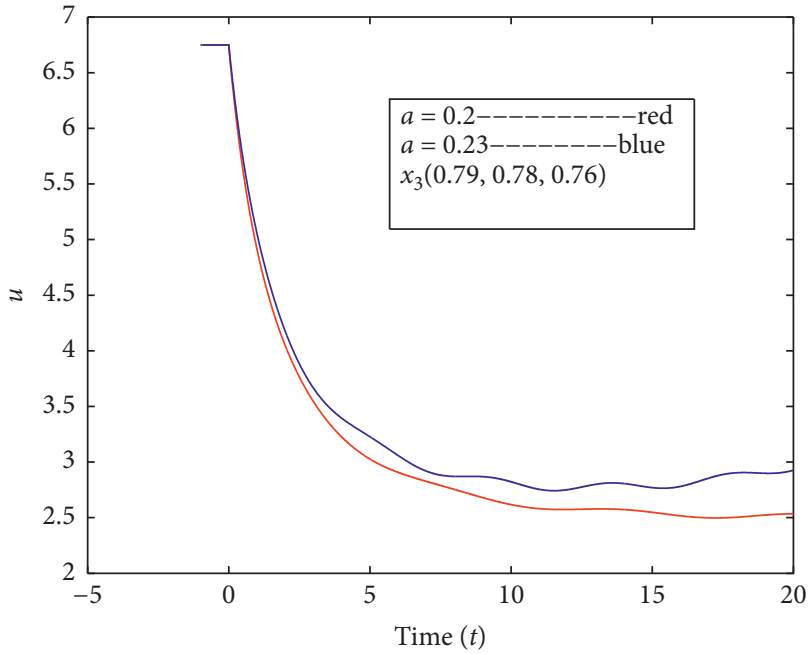

Figure 3: Sectional curves of $u(t, x)$ with $a=0.2$ (red) and $a=0.23$ (blue) at $x_{3}$.

Remark 8. Table 3 provides that due to the loss of natural enemies in a single-species model, the higher the natural population growth rate, the slower the stability of the ecosystem (Figure 3).

Remark 9. Different from [2, 3], the population density boundedness of the species was considered in this study due to the important factor (Remark 4 for details). In Example 1, authors assume $N_{0}=2$ and $M_{0}=10$, and we can see it from Figures $1-3$ that $2<u<10$.

\section{Conclusions}

In this study, critical point theory and variational methods are utilized to derive the unique existence of stationary solution of the single -species model, which is positive and strong. Moreover, the geometric characteristic of saddle point in the mountain pass lemma guarantees that the positive strong stationary solution is the ground state one. Moreover, the method of Lyapunov function yields the global exponential stability of the ground-state classical positive stationary solution which is the unique stationary solution of the ecosystem.

Besides, impulse control reflects the human intervention in the natural ecosystem (e.g., [35], Theorem 3); we may consider the next study on impulsive stabilization of the single-species ecosystem of this study. In addition, we propose mathematical conjectures that ([18], Problem 4) and ([18], Problem 1) may be correct even in the case of the stochastic differential system. In this study, we propose the mathematical conjecture that under suitable conditions, small diffusions that can make the unique stable equilibrium point of the delayed ordinary differential system become multiple stationary solutions of its corresponding partial differential system, even in the case of the stochastic model. Moreover, how to give a global stability invariance criterion of a stochastic model similar as ([18], Corollary 3.4)? This is an interesting problem. Finally, we may consider an interesting application to epidemic control (e.g., [19]). This study is involved in a single-species biological dynamic system. If we investigate the dynamics of a single-species infectious disease model, it is another interesting problem, for the human society may approximately be regarded as a singlespecies model.

\section{Data Availability}

The data used to support the findings of this study are available from the corresponding author upon request.

\section{Conflicts of Interest}

The authors declare that they have no conflicts of interest.

\section{Authors' Contributions}

Ruofeng Rao wrote the article. Quanxin Zhu and Huang Jialin guided the writing of the article and gave their contributions on the revision, being the corresponding authors in charge of the communication of this article. All the authors typed, read, and approved the article.

\section{Acknowledgments}

The authors would like to thank the National Natural Science Foundation of China (61773217) and Chinese Science and Technology Department of Sichuan Province for funding this work through the application basic research project (2020Yj0434) and thank Chengdu Normal University for funding this work through major scientific research projects of Chengdu Normal University (CS19ZDZ01).

\section{References}

[1] L. Chen, X. Meng, and J. Jiao, Biodynamics, Science Press, Beijing, China, 2009.

[2] X. Zou and K. Wang, "A robustness analysis of biological population models with protection zone," Applied Mathematical Modelling, vol. 35, no. 12, pp. 5553-5563, 2011.

[3] W. Ji and G. Hu, "Stability and explicit stationary density of a stochastic single-species model," Applied Mathematics and Computation, vol. 390, Article ID 125593, 2021.

[4] X. Yu, S. Yuan, and T. Zhang, "Persistence and ergodicity of a stochastic single species model with allee effect under regime 
switching," Communications in Nonlinear Science and Numerical Simulation, vol. 59, pp. 359-374, 2018.

[5] Y. Jin, "Analysis of a stochastic single species model with allee effect and jump-diffusion," Advances in Difference Equations, vol. 165, pp. 1-11, 2020.

[6] G. F. Gause, The Struggle for Existence, Williams \& Wilkins, Baltimore, MD, USA, 1935.

[7] J. Huisman, M. Arrayás, U. Ebert, and B. Sommeijer, "How do sinking phytoplankton species manage to persist?" The American Naturalist, vol. 159, no. 3, pp. 245-254, 2002.

[8] R. H. MacArthur and E. O. Wilson, The Theory of Island Biogeography, Princeton University Press, Princeton, NJ, USA, 1967.

[9] J. G. Skellam, "Random dispersal in theoretical populations," Biometrika, vol. 38, no. 1/2, pp. 196-218, 1951.

[10] J. Hofbauer and K. Sigmund, Dynamical Systems and the Theory of Evolution, Cambridge University Press, Cambridge, UK, 1988.

[11] V. Hutson and K. Schmitt, "Permanence and the dynamics of biological systems," Mathematical Biosciences, vol. 111, no. 1, pp. 1-71, 1992.

[12] H. L. Smith and H. Thieme, Dynamical Systems and Population Persistence, American Mathematical Society, Providence, RI, USA, 2011.

[13] R. Rudnicki and K. Pichór, "Influence of stochastic perturbation on prey-predator systems," Mathematical Biosciences, vol. 206, no. 1, pp. 108-119, 2007.

[14] R. Rudnicki, "Long-time behaviour of a stochastic preypredator model," Stochastic Processes and Their Applications, vol. 108, no. 1, pp. 93-107, 2003.

[15] B. Gompertz, "On the nature of the function expressive of the law of human mortality and on a new method of determining the value of life contingencies," Philosophical Transactions of the Royal Society of London, vol. 115, pp. 513-585, 1825.

[16] Y. Zhao and Q. Zhu, "Stabilization by delay feedback control for highly nonlinear switched stochastic systems with time delays," International Journal of Robust and Nonlinear Control, vol. 31, no. 8, pp. 3070-3089, 2021.

[17] R. Rao, "Positive solution for the Dirichlet zero-boundary value problem (In Chinese)," College Mathematics, vol. 26, no. 2, pp. 146-152, 2010.

[18] R. Rao, J. Huang, and X. Li, "Stability analysis of nontrivial stationary solution and constant equilibrium point of reaction-diffusion neural networks with time delays under Dirichlet zero boundary value," Neurocomputing, vol. 445, pp. 105-120, 2021.

[19] R. Rao, "Stability and stabilization of ecosystem for epidemic virus transmission under Neumann boundary value via Impulse control," Preprints, Article ID 2021020197, 2021.

[20] W. Lu, Variational Methods in Differential Equations, Science Press, Beijing, China, 2003.

[21] R. Rao, S. Zhong, and X. Wang, "Stochastic stability criteria with LMI conditions for Markovian jumping impulsive BAM neural networks with mode-dependent time-varying delays and nonlinear reaction-diffusion," Communications in Nonlinear Science and Numerical Simulation, vol. 19, no. 1, pp. 258-273, 2014.

[22] R. Rao, "Global stability of a Markovian jumping chaotic financial system with partially unknown transition rates under impulsive control involved in the positive interest rate," Mathematics, vol. 7, no. 7, 2019.

[23] M. Zhang and Q. Zhu, "Stability analysis for switched stochastic delayed systems under asynchronous switching: a relaxed switching signal," International Journal of Robust and Nonlinear Control, vol. 30, no. 18, pp. 8278-8298, 2020.

[24] U. Humphries, G. Rajchakit, R. Sriraman et al., "An extended analysis on robust dissipativity of uncertain stochastic generalized neural networks with Markovian jumping parameters," Symmetry, vol. 12, no. 6, p. 1035, 2020.

[25] P. Chanthorn, G. Rajchakit, U. Humphries, P. Kaewmesri, R. Sriraman, and C. Lim, "A delay-dividing approach to robust stability of uncertain stochastic complex-valued hopfield delayed neural networks," Symmetry, vol. 12, no. 5, 2020.

[26] U. Humphries, G. Rajchakit, P. Kaewmesri et al., "stochastic memristive quaternion-valued neural networks with time delays: an analysis on mean square exponential input-to-state stability," Mathematics, vol. 8, no. 5, 2020.

[27] P. Chanthorn, G. Rajchakit, J. Thipcha et al., "Robust stability of complex-valued stochastic neural networks with timevarying delays and parameter uncertainties," Mathematics, vol. 8, no. 5, p. 742, 2020.

[28] M. S. Ali, M. Usha, Q. Zhu, and S. Shanmugam, "Synchronization analysis for stochastic T-S fuzzy complex networks with Markovian jumping parameters and mixed time-varying delays via impulsive control," Mathematical Problems in Engineering, vol. 2020, p. 9739876, 2020.

[29] F. Kong, Q. Zhu, R. Sakthivel, and A. Mohammadzadeh, "Fixed-time synchronization analysis for discontinuous fuzzy inertial neural networks with parameter uncertainties," Neurocomputing, vol. 422, pp. 295-313, 2021.

[30] W. Cao and Q. Zhu, "Razumikhin-type theorem for pth exponential stability of impulsive stochastic functional differential equations based on vector Lyapunov function," Nonlinear Analysis: Hybrid Systems, vol. 39, p. 100983, 2021.

[31] F. Kong and Q. Zhu, "New fixed-time synchronization control of discontinuous inertial neural networks via indefinite Lyapunov-Krasovskii functional method," International Journal of Robust and Nonlinear Control, vol. 31, no. 2, pp. 471-495, 2020.

[32] F. Kong, Q. Zhu, and T. Huang, "New fixed-time stability lemmas and applications to the discontinuous fuzzy inertial neural networks," IEEE Transactions on Fuzzy Systems, p. 1, 2020.

[33] R. Song, B. Wang, and Q. Zhu, "Delay-dependent stability of nonlinear hybrid neutral stochastic differential equations with multiple delays," International Journal of Robust and Nonlinear Control, vol. 31, no. 1, pp. 250-267, 2021.

[34] L. Gao, Z. Cao, M. Zhang, and Q. Zhu, "Input-to-state stability for hybrid delayed systems with admissible edge-dependent switching signals," Journal of the Franklin Institute, vol. 357, no. 13, pp. 8823-8850, 2020.

[35] R. Rao, Q. Zhu, and K. Shi, "Input-to-State stability for impulsive gilpin-ayala competition model with reaction diffusion and delayed feedback," IEEE Access, vol. 8, pp. 222625-222634, 2020.

[36] X. Yang and Q. Zhu, "Stabilization of stochastic functional differential systems by steepest descent feedback controls," IET Control Theory \& Applications, vol. 15, no. 6, pp. 805-813, 2021.

[37] H.-S. Ding and Q. He, "Existence and asymptotic behavior of positive ground state solutions for nonlinear Kirchhoff problems," Communications in Nonlinear Science and $\mathrm{Nu}$ merical Simulation, vol. 90, Article ID 105369, 2020.

[38] S. Tian, "Non-degeneracy of the ground state solution on nonlinear Schrödinger equation," Applied Mathematics Letters, vol. 111, Article ID 106634, 2021. 
[39] X. Chen, X. Wu, and C. Tang, "existence of a positive ground state solution for a class of asymptotically 3-linear Kirchhofftype equations (in Chinese)," Journal of Southwest China Normal University(Natural Science Edition), vol. 44, no. 4, pp. 22-25, 2019.

[40] Y. Li, G. Li, and C. Tang, "Existence and concentration of ground state solutions for Choquard equations involving critical growth and steep potential well," Nonlinear Analysis, vol. 200, Article ID 111997, 2020.

[41] R. Rao and S. Zhong, "Impulsive control on delayed feedback chaotic financial system with Markovian jumping," Advances in Difference Equations, vol. 50, 2020.

[42] X.-X. Liao and J. Li, "Stability in Gilpin-Ayala competition models with diffusion," Nonlinear Analysis: Theory, Methods \& Applications, vol. 28, no. 10, pp. 1751-1758, 1997.

[43] L. Bai and K. Wang, "Gilpin-Ayala model with spatial diffusion and its optimal harvesting policy," Applied Mathematics and Computation, vol. 171, no. 1, pp. 531-546, 2005.

[44] Y. Liu and Y. Tao, "Dynamics in a parabolic-elliptic twospecies population competition model with cross-diffusion for one species," Journal of Mathematical Analysis and Applications, vol. 456, no. 1, pp. 1-15, 2017.

[45] K. Ding and Q. Zhu, "Fuzzy intermittent extended dissipative control for delayed distributed parameter systems with stochastic disturbance: a spatial point sampling approach," IEEE Transactions on Fuzzy Systems, p. 1, 2021.

[46] X. Liu and Q. Zhu, "Stochastically globally exponential stability of stochastic impulsive differential systems with discrete and infinite distributed delays based on vector Lyapunov function," Complexity, vol. 2020, Article ID 7913050, 16 pages, 2020 . 